\title{
Computer control for operant oriented laboratories
}

\author{
RICHARD A. MARTIN and ROBERT J. CONNER \\ Indiana University, Bloomington, Indiana 47401.
}

\begin{abstract}
A process control procedure using base rate polling within interrupt driven routines, segmentation of time dependent events, and a branch table execution structure was developed for an operant oriented behavioral pharmacology laboratory. Efficient independent control and analysis for eight experimental chambers was obtained utilizing the similarities between various operant techniques.
\end{abstract}

\section{HISTORY}

The behavioral pharmacology laboratory at Indiana University has been using a process control type software package for the instrumentation of an expanding operant oriented program of animal experimentation. Over the past 5 years this system has allowed the instrumentation of increasingly complex paradigms with no major revision and only minor changes after 2 years of operation. The software is implemented within the Multiprogramming Executive operating system (MPX) of an IBM 1800 located in the department; however, the structure and programming techniques are not limited to that installation. Convenience, efficiency, flexibility, and the lack of software problems have contributed to a package structure well suited to our laboratory operation.

\section{APPLICATION}

The package is used for animal (primarily rat) learning and memory applications oriented around discrete trial operant behavioral techniques [Heise, G. A. (in press); Krank, M., Christoph, G., \& Leinwand, S. (Note 2)]. Typically, a trial/intertrial interval sequence is presented. One or more discriminative stimuli indicate a trial and either a response or the passage of a predetermined interval terminates the trial and initiates the next intertrial interval. For these types of procedures, whether discrete trial or free operant, it is convenient to describe the process as a finite set of linked "intervals" or "states" marked in time. Stimulus presentation and consequences of subject responses are simply control decisions laid upon the linked-state structure. Due to the limited capabilities of the animal subjects and our difficulty in coping with complex and often confounded stimulus-response networks, the relatively small number of discrete time-event relationships which can be reasonably expected allows the imposition of restrictions

This work was supported by National Institutes of Mental Health Grant PHS PO1 MH14658 awarded to George A. Heise. The IBM 1800 installation was supported by Public Health Service Grant PHS R01 MH16817 awarded to Frank Restle. Requests for reprints or related information should be sent to Richard A. Martin, Department of Psychology, Indiana University, Bloomington, Indiana 47401 . on the requirements of the control system. Our experience has indicated that: (a) three active response types (actuation of three different levers, or two levers and chain pull, etc.) are generally adequate for the assessment of an individual animal subject's behavior; (b) eight stimulus drivers (a mixture of cue lights, tones, dispensing solenoids, etc.) provide a sufficient number of environmental stimuli; (c) 14 states allow the necessary description of extremely complex paradigms.

The above limits provided a convenient and efficient framework upon which the software was structured. Also of importance was the fact that our experimental procedures extend over a period of several months and are not often changed. A further design consideration was the availability of hardware, memory, and interactive software (MPX) at the IBM 1800 installation. The final package consists of a FORTRAN initialization and setup section, a FORTRAN data analysis section, and an assembly language control routine. This control routine is the heart of the system and utilizes a buffered FORTRAN disk data transfer.

\section{STRUCTURE}

Many arbitrary definitions concerning short periods of time are made in the design of any real-time control and analysis system. In the instrumentation of most experimental procedures, the criterion used to judge the importance of those definitions is often no more than the pragmatism of the experimenter. The relative consistency in a short interval timing definition is therefore fundamental to adequate experimental control. This definition specifies a resolution at which the process is to be viewed for control and analysis.

The independent computer control of experimental procedures which involve both time and response dependent functions necessitates an interrupt structure and a clocking network. Each unit (in our case one of eight experimental chambers) must be serviced within its own time frame and therefore requires the use of at least one clock which is controlled by the Executive system. Although interrupt servicing must be immediate, action based on that service may be arbitrarily delayed for a 


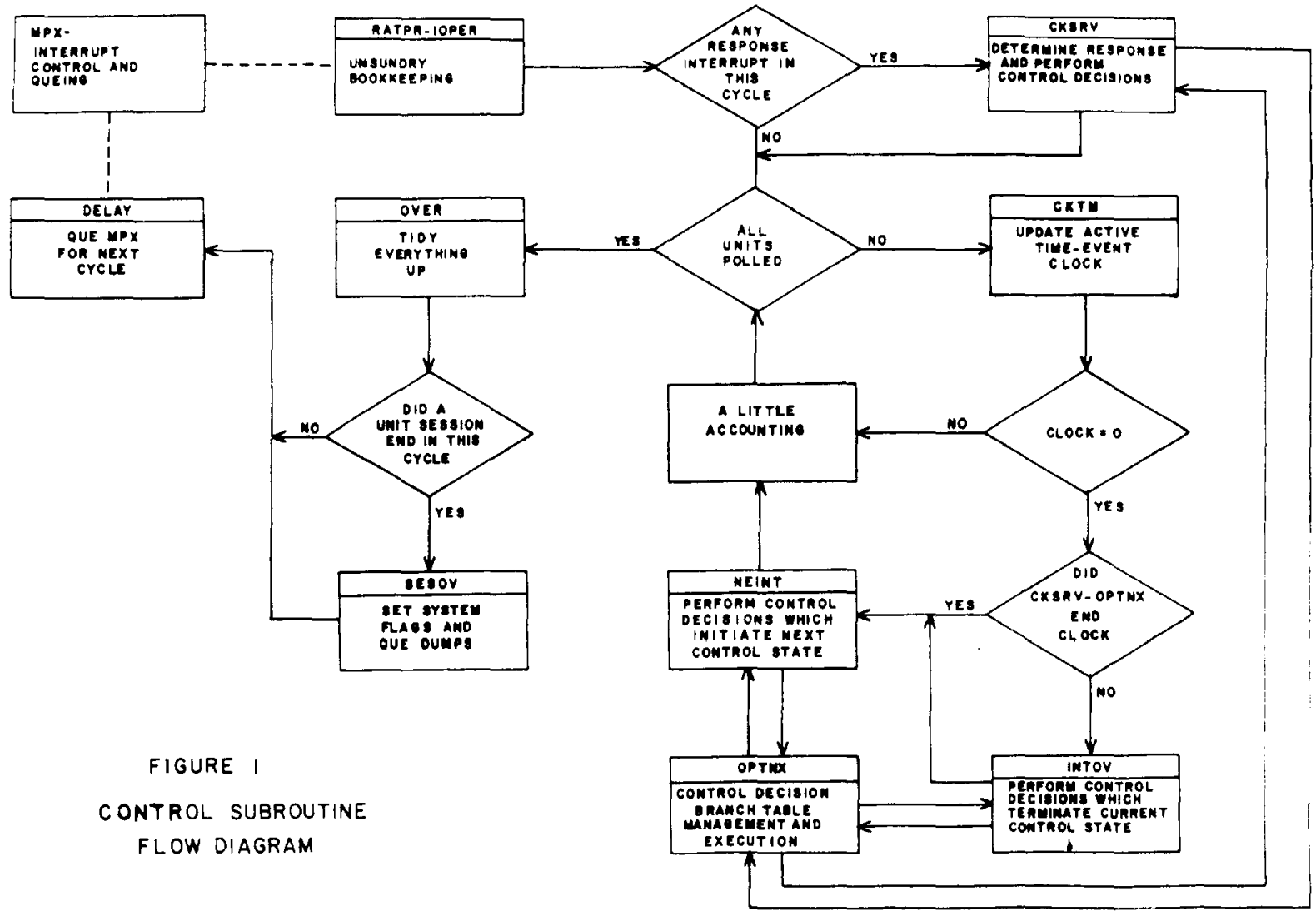

period of time consistent with the experimental demands and equipment capabilities.

In order to generate sufficient numbers of independent (yet synchronized by the Executive system) clocks, to allow necessary processor time for all system demands, and yet service interrupts within allowable error, the control procedure routine was structured as a subroutine called by the Executive on a .1-sec clock interrupt cycle. Interrupt service, removal of system flag, is initially done by the Executive and the results of that service, i.e., the need for control decisions, are examined at the .1-sec rate by the control procedure subroutine (however, some types of immediate decisions can be done in the Executive interrupt service routine). After the servicing of unit generated requests (serviced interrupts caused by animal responses), each unit is polled for clocking activity. Active clocks are updated and tested for service based upon a zero clock value. In addition, each unit is provided with a 1 -min clock which is used to update a unit dependent session termination clock. (This clock forces termination of the session after the expiration of its preset value if the session counter has not terminated the session.) The cyclic interrupt serves to synchronize the MPX system, unit generated interrupts, and the strictly time dependent control functions.

Figure 1 provides a simplified flow diagram of the procedure logic. Based upon IBM 1800 instruction execution times, the minimum loop time with eight active units is approximately 765 microsec. An animal initiated response which causes the termination of one state and the beginning of another requires an average of $4.2 \mathrm{msec}$. The worst case timing for a single cycle is approximately $92 \mathrm{msec}$, i.e., under the 100 -msec cycle limit and within the range of resolution.

For the purpose of control, a "discrete time-event relationship" called an "option" was defined as any instance that caused a change in the static condition of any particular state. These changes can be divided into three basic classes: external control (stimulus presentation), monitor functions (data collection), and internal control (mechanisms for flow between states). Although experimental units utilize the various discrete time-event relationships in different sequential orders, the code required to execute any particular option is not redundant within the control scheme since each unit was provided with unique dynamic parameter storage and a shared static table area which linked the options via indices maintained as schedule dependent. This technique (a crude and incomplete form of reentrancy) allowed considerable memory savings and greatly simplified the compilation of the unitized control procedures. Each option was thus a quasireentrant subroutine called from an appropriate state control macro. In Figure 1, note that the macro titled OPTNX is referenced by three separate state control areas. CKSRV manages the response dependent control and may or may not include state termination. INTOV handles state 
terminations that are strictly time dependent and NEINT performs the control decisions necessary to initiate a new state.

The OPTNX macro manages the sequential execution of the discrete options using a branch table whose indices are a function of the current state and the source of the call. The values of the table are address indices locating a specific coded time-event relationship. Referring to Figure 2, Array A (eight elements) contains the starting address of the $B$ table associated with the particular schedule which is currently linked to the experimental unit. (At present, the package allows the operation of four distinct schedules.) The B table is organized as a two-dimensional array indexed by state number (i) and state control function (t). The state numbers range from 0 to 13 and the state control functions are of types "s" (start or initiate state), "o" (time-out or nonresponse), and 1,2,3 (range of possible active responses). Each entry in $B$ is the starting address of a list (contained in $\mathrm{C}$ ) whose elements are pointers for specific time-event options. Each list has a limit of 16 options, packed two per word. The pointers are displacements for Table $\mathrm{D}$, whose contents are in turn displacements for the location of the absolute entry address for the option execution code. Although this scheme is complex, experience has proven its worth. Options may be added or deleted from the main
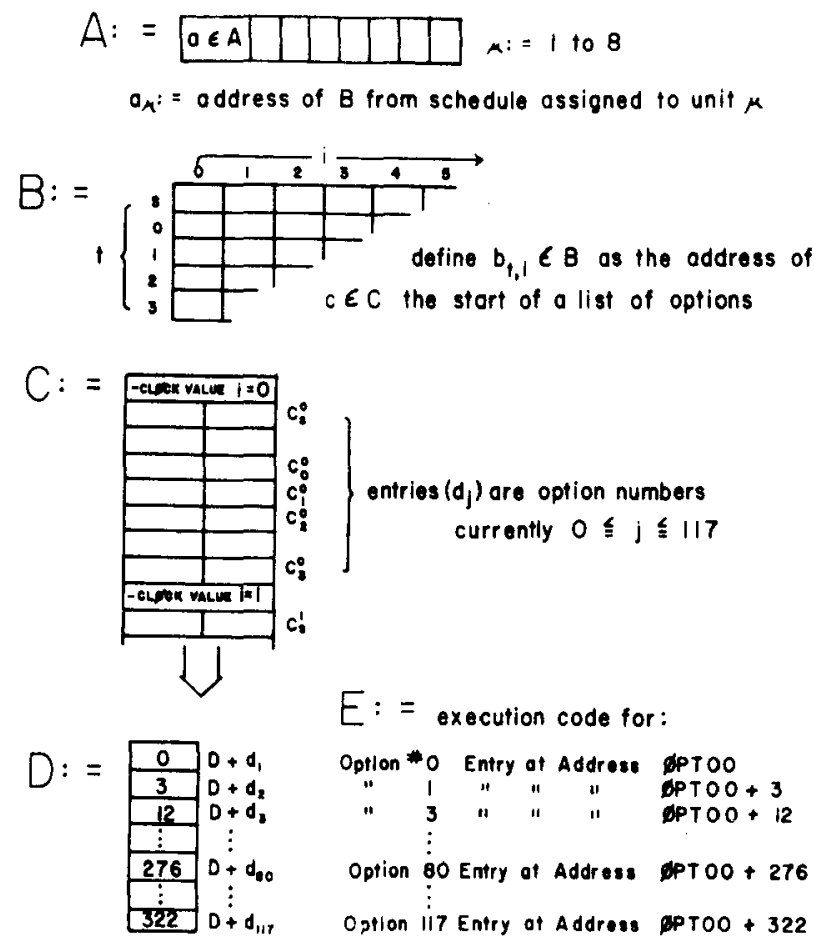

FIGURE 2

OPTNX Branch Scheme
Table 1

Currently Available Options

\begin{tabular}{|c|c|c|}
\hline Class & $\begin{array}{c}\text { Option } \\
\text { Number } \\
\text { (OPN) }\end{array}$ & Function \\
\hline Internal & 0 & Auxillary list terminator \\
\hline Internal & 1 & End time interval with response \\
\hline Monitor & 2 & Da ta dump to sequential file \\
\hline Monitor & 3 & Decrement session counter \\
\hline Internal & 4 & Increment Sequence 1 index \\
\hline Internal & 5 & Increment Sequence 2 index \\
\hline Internal & 6 & $\begin{array}{l}\text { Pull even-odd value of Seq } 1 \text { at index } \\
\text { and store } Q_{(0,1)} \text { at "NXTRT" }\end{array}$ \\
\hline Internal & 7 & $\begin{array}{l}\text { Pull } 0-3 \text { value of Seq } 1 \text { at index and } \\
\text { store } Q_{(2.5)} \text { at "NXTRT" }\end{array}$ \\
\hline Internal & 8 & $\begin{array}{l}\text { Pull } 0-4 \text { value of Seq } 2 \text { at index and } \\
\text { store } Q_{(6.11)} \text { at "N3INT" }\end{array}$ \\
\hline Internal & $9-22$ & NXINT $=$ OPN $-9:$ Value $0-13$ \\
\hline Internal & $23-36$ & N2INT $=$ OPN-23: Value $0-13$ \\
\hline Internal & $37-50$ & N3INT $=$ OPN-37: Value $0-13$ \\
\hline Internal & $51-64$ & NXTRT $=$ OPN $-51:$ Value $0-13$ \\
\hline Internal & 65 & NXINT $=$ NXTRT \\
\hline Internal & 66 & NXINT = N2INT \\
\hline Internal & 67 & $\mathrm{NXINT}=\mathrm{N} 3 \mathrm{INT}$ \\
\hline Internal & 68 & NXTRT $=$ N2INT \\
\hline Internal & 69 & NXTRT $=$ N3INT \\
\hline Internal & 70 & $\mathrm{~N} 2 \mathrm{INT}=\mathrm{N} 3 \mathrm{INT}$ \\
\hline External & 71 & Stop bit flash \\
\hline External & 72 & Set Output Bit 8 for $.1 \mathrm{sec}$ \\
\hline External & 73 & Set Output Bit 7 for $.1 \mathrm{sec}$ \\
\hline External & $74-81$ & Flash Output Bit OPN-73 at .1-sec rate \\
\hline External & $82-89$ & Set Output Bit OPN-81 \\
\hline External & $90-97$ & Reset Output Bit OPN-89 \\
\hline Monitor & $98-117$ & $\begin{array}{l}\text { Increment cumulative counter OPN-97 } \\
\text { by one }\end{array}$ \\
\hline
\end{tabular}

program by first changing the assembly code in $\mathrm{E}$ and then updating $D$ to reflect address displacement changes. Any particular schedule is defined by the set $[B, C]$. A practical limit of 185 words was placed on the size of $C$; therefore, the limit of the complexity of the schedule encoding is represented by:

$$
11 \leqslant \# \mathrm{I}+\sum_{\mathrm{i}=0}^{\# \mathrm{I}} \sum_{\mathrm{t}=5}^{3} \frac{\mathrm{OPT}_{\mathrm{i}, \mathrm{t}}}{2} \leqslant 185
$$

where \#I is the number of states used and OPT $i, t$ is the number of options used at $t$ for $i \epsilon I$, the set of utilized states, such that $2 \leqslant O P T_{i, t} \leqslant 16$.

By using two tables to define the schedule, $[B, C]$, instead of one table (indexed by $i$ and $t$ with packed entries $\mathrm{d}_{\mathrm{j}}$ ) and by placing a limit on the size of $\mathrm{C}$, a savings of 319 words was realized for each schedule loaded to memory (a savings of 1,276 words if four schedules were in operation simultaneously).

Tables $\mathrm{B}$ and $\mathrm{C}$ are built with the first loading of $\mathrm{a}$ schedule and reside on the disk for future reference. The entires into $A$ are made when $[B, C]$ is loaded into memory at a session initiate.

Referring to Table 1, external control is provided by Options 71 through 97 (see Table 2 for current hardware assignments). Monitor functions are assumed by Options 
Table 2

Current Hardware Assignments

\begin{tabular}{lll}
\hline & 1 & Left lever or key \\
Response & 2 & Right lever or key \\
Line & 3 & Center key or touch rod \\
& 1 & Right cue light \\
& 2 & Center cue light \\
& 3 & Left cue light \\
Output & 4 & 3000-Hz tone \\
Line & 5 & 1000-Hz tone \\
& 6 & Houselight \\
& 7 & Shock generator or touch rod solenoid \\
& 8 & Liquid reinforcement solenoid \\
\hline
\end{tabular}

2 and 3 and 98 through 117 . The latter set of options can be viewed as simple cumulative counters and Option 3 as a predetermined session counter for session termination. Option 2 places a data word containing the current interval value (0-13), current response type $(0,1$, $2,3)$, and current interval clock value ( -511 to 0 ) onto a sequential unit dependent file on the disk. In this manner, an entire session may be saved event by event for analysis. The data word format is: interval-Bits $0-3$, response type-Bits 4 and 5, clock-Bits 6-15.

The remaining options provide the internal control of state flow. The control procedure replaces the value of CRINT(u), an interval variable, with NXINT(u), a user variable, to obtain the value for a new state and references $B$ at bCRINT,s to initiate that state. Aside from this single automatic control decision, all other state linkage is determined by options from Table 1 entered into C. NXTRT, N2INT, and N3INT should be considered temporary storage locations. Option 1 allows immediate response dependent state termination and the subsequent bypass of the normal state termination option list execution. Options 4 through 8 allow for fixed sequences of digits to be utilized in the state flow decision process. For descriptive purposes, assume
Vector $\mathbf{Q}$ of 11 elements is a sequential file with pointers fixed at $Q_{0}, Q_{2}$, and $Q_{6}$. Option 6 references $Q$ beginning at $Q_{0}$ and indexes by 0 or 1 , depending upon the respective even- or odd-valued number encountered in the first fixed sequence. Option 7 indexes from $Q_{2}$ with the value of the fixed sequence at the index established by Option 4 . Option 8 indexes from $Q_{6}$ in a like manner using the second fixed sequence. In each case, the value of $\mathrm{Q}$ is stored at the named variable label.

The options enumerated in Table 1 have been in use for over 2 years and have so far met all experimental needs. The experimenter specifies the contents of $[B, C]$ by creating a schedule table, $S$, and specifies initial variable values in a unit dependent parameter vector, $P$. An $S$ consists of a header card giving the schedule number and $Q$ followed by a set of six cards for each desired state. Card 1 gives the state number, state duration (a negative value at resolution base), and the number of options for each $t$ (always an even number between 2 and 16). The subsequent cards in the set specify the options for each $t$ in the order $s, 0,1,2,3$. A $P$ consists of a unit header card and a vector card containing the following initialization values: unit number, session duration maximum time (in minutes), preset session counter value, value of current clock, CRINT, NXINT, N2INT, N3INT, NXTRT, schedule number, and end method ( 1 indicates session counter termination). Table 3 gives a simple procedure used to test a unit's hardware and Table 4 is one of the more complex schedules yet used. Both are left for the reader's enjoyment.

The development of $[\mathrm{S}, \mathrm{P}]$ forces the experimenter into a detailed examination of the proposed experimental procedure. We feel this examination process has extended the scope with which paradigms were viewed and has allowed a more creative approach to experimentation. The control procedure subroutine is

Table 3

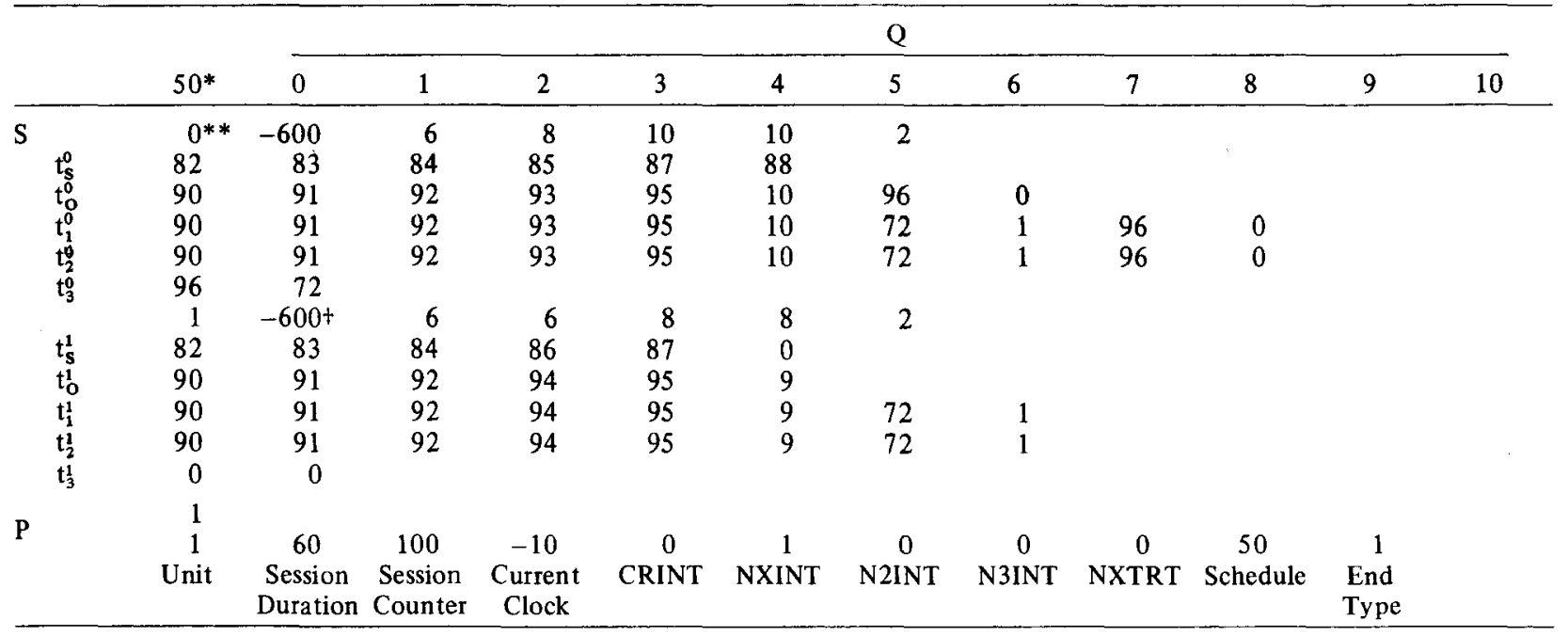


Table 4

Experimenter Coding for Branch Table Creation: Two-Trial Delayed Response With Extra-Trial Events

\begin{tabular}{|c|c|c|c|c|c|c|c|c|c|c|c|c|c|c|c|c|c|c|c|}
\hline 91 & 0 & 1 & 2 & 3 & 4 & 5 & 7 & 7 & 8 & & & 98 & 0 & & & & & & \\
\hline 0 & -50 & 6 & 8 & 10 & 8 & 2 & & & & & & 8 & -40 & 2 & 4 & 2 & 2 & 6 & \\
\hline 86 & 95 & 83 & 0 & & & & & & & & & 88 & 18 & & & & & & \\
\hline 111 & 94 & 20 & 91 & 90 & 92 & 2 & 0 & & & & & 0 & 0 & & & & & & \\
\hline 109 & 94 & 15 & 72 & 90 & 92 & 2 & 91 & 1 & 0 & & & 107 & 2 & & & & & & \\
\hline 110 & 94 & 20 & 91 & 90 & 92 & 2 & 1 & & & & & 107 & 2 & & & & & & \\
\hline 98 & 0 & & & & & & & & & & & 103 & 96 & 72 & 2 & 19 & 0 & & \\
\hline 1 & -50 & 6 & 8 & 8 & 10 & 2 & & & & & & 9 & -800 & 2 & 4 & 2 & 2 & 6 & \\
\hline 85 & 95 & 83 & 0 & & & & & & & & & 88 & 0 & & & & & & \\
\hline 116 & 93 & 20 & 91 & 90 & 92 & 2 & 0 & & & & & 1.13 & 19 & 2 & 96 & & & & \\
\hline 115 & 93 & 20 & 91 & 90 & 92 & 2 & 1 & & & & & 107 & 2 & & & & & & \\
\hline 114 & 93 & 15 & 72 & 90 & 92 & 2 & 91 & 1 & 0 & & & 107 & 2 & & & & & & \\
\hline 98 & 0 & & & & & & & & & & & 108 & 96 & 72 & 19 & 2 & 1 & & \\
\hline 2 & -50 & 2 & 6 & 6 & 10 & 2 & & & & & & 10 & -20 & 2 & 4 & 4 & 4 & 4 & \\
\hline 83 & 0 & & & & & & & & & & & 0 & 0 & & & & & & \\
\hline 101 & 91 & 20 & 2 & 50 & 0 & & & & & & & 65 & 2 & 95 & 0 & & & & \\
\hline 100 & 91 & 20 & 2 & 1 & 50 & & & & & & & 117 & 19 & 2 & 1 & & & & \\
\hline 99 & 91 & 20 & 2 & 72 & 5 & 5 & 3 & 1 & 8 & & & 117 & 19 & 2 & 1 & & & & \\
\hline 98 & 0 & & & & & & & & & & & 98 & 19 & 2 & 1 & & & & \\
\hline 3 & -50 & 2 & 6 & 10 & 6 & 2 & & & & & & 11 & -190 & 2 & 2 & 2 & 2 & 2 & \\
\hline 83 & 0 & & & & & & & & & & & 87 & 0 & & & & & & \\
\hline 101 & 91 & 20 & 2 & 50 & 0 & & & & & & & 21 & 6 & & & & & & \\
\hline 99 & 91 & 20 & 2 & 72 & 5 & 4 & 3 & 1 & 8 & & & 102 & 0 & & & & & & \\
\hline 100 & 91 & 20 & 2 & 1 & 50 & & & & & & & 102 & 0 & & & & & & \\
\hline 98 & 0 & & & & & & & & & & & 98 & 0 & & & & & & \\
\hline 4 & -50 & 2. & 6 & 8 & 12 & 2 & & & & & & 12 & -10 & 2 & 8 & 4 & 4 & 2 & \\
\hline 106 & 91 & 93 & 20 & 2 & 50 & & & & & & & 0 & 0 & & & & & & \\
\hline 105 & 91 & 93 & 20 & 2 & 1 & 50 & 0 & & & & & 65 & 95 & 90 & 91 & 92 & 93 & 94 & 7 \\
\hline 104 & 91 & 93 & 20 & 2 & 72 & 5 & 4 & 3 & 1 & 8 & & 102 & 21 & 1 & 0 & & & & \\
\hline 98 & 0 & & & & & & & & & & & 102 & 21 & 1 & 0 & & & & \\
\hline 5 & -50 & 2 & 6 & 12 & 8 & 2 & & & & & & 98 & 0 & & & & & & \\
\hline 83 & 86 & & & & & & & & & & & 13 & -40 & 2 & 2 & 2 & 2 & 2 & \\
\hline 106 & 91 & 94 & 20 & 2 & 50 & & & & & & & 0 & 0 & & & & & & \\
\hline 104 & 91 & 94 & 20 & 2 & 72 & 5 & 4 & 3 & 1 & 8 & 0 & 19 & 0 & & & & & & \\
\hline 105 & 91 & 94 & 20 & 2 & 1 & 50 & 0 & & & & & 0 & 0 & & & & & & \\
\hline 98 & 0 & & & & & & & & & & & 0 & 0 & & & & & & \\
\hline 6 & -40 & 2 & 2 & 2 & 2 & 2 & & & & & & 98 & 0 & & & & & & \\
\hline 87 & 0 & & & & & & & & & & & & & & & & & & \\
\hline 67 & 2 & & & & & & & & & & & & & & & & & & \\
\hline 102 & 2 & & & & & & & & & & & & & & & & & & \\
\hline 102 & 2 & & & & & & & & & & & & & & & & & & \\
\hline 98 & 0 & & & & & & & & & & & & & & & & & & \\
\hline 7 & -40 & 2 & 4 & 2 & 2 & 4 & & & & & & & & & & & & & \\
\hline 0 & 0 & & & & & & & & & & & & & & & & & & \\
\hline 19 & 2 & 94 & 0 & & & & & & & & & & & & & & & & \\
\hline 112 & 2 & & & & & & & & & & & & & & & & & & \\
\hline 112 & 2 & & & & & & & & & & & & & & & & & & \\
\hline 1 & & & & & & & & & & & & & & & & & & & \\
\hline 1 & 120 & 100 & -100 & 11 & 0 & 0 & 8 & 3 & 91 & 1 & & & & & & & & & \\
\hline
\end{tabular}

completely predictable and allows a total recreation of an experimental session (i.e., Option 2) for analysis purposes. Experimenter coding errors have been easily discovered at initial testing and corrections have been made quickly. The experimenter is not required to learn a complex computer language and need only reference Tables 1 and 2 to code a paradigm.

\section{SYSTEM}

The host IBM 1800 installation consists of a $32 \mathrm{~K}$ CPU with a 2315 dual disk drive, 1442 card reader/punch and a 1443 line printer. The MPX operating system
(MPX V3M3) has a 17.3K Executive, seven partitions $(2 \mathrm{~K}, .9 \mathrm{~K}, .6 \mathrm{~K}, .8 \mathrm{~K}, 1 \mathrm{~K}, 1.1 \mathrm{~K})$ and 8,468 words of variable core (VCORE) (IBM 1800 Multiprogramming Executive Operating System Programmer's Guide, Order Number GC26-3720-5, Note 1). The last block is used for all low priority and batch type processing. The Executive contains two routines that handle the interrupts of current interest and places status flags in a common area available to all partitions and VCORE. The assembly language control subroutine resides in the seventh partition and is currently 920 words in length, of which 329 words are option execution code. Variable and table storage required 1,721 words allocated as: 
1,020 for [B,C] tables ( 4 sets), 120 for the single D table, 320 for data buffers and cumulative counters, and 261 for all other variables. This configuration is adequate for the independent control of eight experimental units.

A set of FORTRAN routines which execute in VCORE comprise the initialization and setup portion of the package. These routines perform the $[S, P]$ to $[B, C]$ transformations and manage a disk file which contains the information necessary to initiate any 1 of 16 disk resident schedules. With these routines, it is often the case that all setup and initiation is predefined for an entire experiment and only the unit and schedule must be selected to actually begin a session. Another set of FORTRAN routines, again executed in VCORE, operate in conjunction with the control subroutine to dump data buffers to unit dependent disk files. Each file contains header information, Option 2 data and cumulative count data for sequential sessions. Each has a capacity of 2,240 words.

The operation of the program package so far described (initialization and setup, control procedure, and data dump) is strictly the responsibility of the laboratory systems analyst. With the exception of the $[S, P]$ structure development and the calling to execution of resident routines, the experimenter does not interact with or modify the package. This feature has in part contributed to the continued success of the system.

Data analysis presents a reversed situation. Experimenters are required to structure their data so as to fit an existing reduction scheme or to write the necessary programs to handle their unique data form or analysis. This is most typically accomplished by utilizing a standard routine for decoding header and cumulative counter information and coupling this with a specific driver to decode the event by event data. All data analysis is done in the batch mode on a daily basis.

\section{EXTENSIONS}

Although the package as outlined here requires a rather sophisticated support structure, the basic techniques described can be implemented to great advantage in a much smaller system. If one is willing to load $[B, C]$ tables from paper tape and limit the size of data buffers to available memory, there is no reason why 8 or even 16 units could not be run with as little as $4 \mathrm{~K}$. The great disadvantage of such a system is the inability to store large quantities of event by event data and effectively to analyze that data. This capability has become essential to our experimental program and hence we have used the larger system.

The power and flexibility of the control subroutine can be increased in several ways. First, the D table and E code section could be incorporated as independent of the control subroutine and thus allow simple option addition, deletion, or modification. In fact, entire sets of $[D, E]$ could be disk resident for specific sets $[B, C]$. Second, the $[B, C]$ sets could be expanded to include a Table B'. This table would index the $b_{i, s}$ address for each $\mathrm{i} \in \mathrm{I}$. B could then be expanded to include a variable number of $t$ assignable to any $i \epsilon$. In addition, the $[\mathrm{S}, \mathrm{P}]$ to $\left[\mathrm{B}^{\prime}, \mathrm{B}, \mathrm{C}\right]$ transformation could be done so as to eliminate redundancies in $\mathrm{C}$ when the options for $b_{i, t}$ and $b_{j, t}$ are the same for $i \neq j$. Another useful change concerns the dynamic memory allocation of the tables within the package. This, however, requires an operating system capable of utilizing any memory savings realized as a result of that dynamic allocation. Since the internal flag coding for the control routine is packed, the inclusion of another eight units is a simple matter necessitating trivial coding changes and the addition of approximately 200 words of variable parameter storage.

These extensions, which increase the power and flexibility of the package, are presently being included in a new version of the package being installed on our TI980A computer system.

\section{REFERENCE NOTES}

1. International Business Machines Corporation, Data Processing Division, 1133 Westchester Avenue, White Plains, New York. IBM 1800 Multiprogramming Executive Operating System Programmer's Guide. Order Number GC26-3270-5.

2. Krank, M., Christoph, G., \& Leinwand, S. Proactive influences on spatial alternation in the rat. Presented at the Annual Meeting of the Midwestern Psychological Association, May 2-4, 1974.

\section{REFERENCE}

Heise, G. A. Discrete trial analy sis of drug action. Federation Proceedings, 1975, in press. 